\title{
Casimir effect in $(2+1)$-dimensional Yang-Mills theory as a probe of the magnetic mass
}

\author{
Dimitra Karabali ${ }^{1, *}$ and V.P. Nair ${ }^{2, \dagger}$ \\ ${ }^{1}$ Department of Physics and Astronomy, Lehman College of the CUNY, Bronx, New York 10468, USA \\ ${ }^{2}$ Physics Department, City College of the CUNY, New York, New York 10031, USA
}

(Received 28 August 2018; published 20 November 2018)

\begin{abstract}
We consider the Casimir effect in a gauge-invariant Hamiltonian formulation of non-Abelian gauge theories in $(2+1)$ dimensions, for an arbitrary gauge group. We show that the result is in good agreement with recent lattice simulations. We also argue that the Casimir effect may be viewed as a good probe of magnetic screening effects in $(3+1)$-dimensional gauge theories at high temperatures.
\end{abstract}

DOI: 10.1103/PhysRevD.98.105009

\section{INTRODUCTION}

Yang-Mills gauge theories in two spatial dimensions can be viewed as a guiding model for the more realistic, but also more complicated, $(3+1)$-dimensional gauge theories. The $(2+1)$-dimensional theories have nontrivial dynamical content and propagating degrees of freedom making them a better model than Yang-Mills theories in $(1+1)$ dimensions, yet they are still somewhat more amenable to mathematical analysis compared to their $(3+1)$ dimensional counterparts. The Euclidean 3-dimensional theory, the Wick-rotated version of the $(2+1)$-dimensional theory, can also be of direct relevance to the high temperature limit of the $(3+1)$-dimensional theory [1]. In particular, the mass which appears as a propagator mass in $(2+1)$ dimensions can be taken as the high temperature value of the magnetic screening mass. With these motivations, for many years, we have been pursuing a Hamiltonian approach to the nonperturbative aspects of Yang-Mills theories in $(2+1)$ dimensions [2-4]. This article will be in the nature of continued work along these lines, focusing on the Casimir effect in Yang-Mills theories in $(2+1)$ dimensions. This was also inspired by the recent lattice simulations of the Casimir effect for the $S U(2)$ gauge theory reported in [5]. We will argue that the Casimir effect in the $(2+1)$-dimensional Yang-Mills theory can be viewed as a probe of the magnetic mass in the pure QCD plasma in $(3+1)$ dimensions at high temperatures. This will also furnish a calculation for a general gauge group

\footnotetext{
dimitra.karabali@lehman.cuny.edu

†pnair@ccny.cuny.edu
}

Published by the American Physical Society under the terms of the Creative Commons Attribution 4.0 International license. Further distribution of this work must maintain attribution to the author(s) and the published article's title, journal citation, and DOI. Funded by SCOAP ${ }^{3}$. which can, hopefully, be tested in lattice simulations in the near future.

We begin with a brief recapitulation of the salient points of our Hamiltonian analysis. We considered the $A_{0}=0$ gauge, with the spatial components of the gauge potentials parametrized as

$$
\begin{aligned}
& A_{z}=\frac{1}{2}\left(A_{1}+i A_{2}\right)=-\partial M M^{-1}, \\
& A_{\bar{z}}=\frac{1}{2}\left(A_{1}-i A_{2}\right)=M^{\dagger-1} \bar{\partial} M^{\dagger}
\end{aligned}
$$

Here we use complex coordinates $z=x_{1}-i x_{2}, \bar{z}=x_{1}+i x_{2}$ with $\partial=\frac{1}{2}\left(\partial_{1}+i \partial_{2}\right), \quad \bar{\partial}=\frac{1}{2}\left(\partial_{1}-i \partial_{2}\right)$, and $M$ is an element of the complexified group $G^{\mathbb{C}}$; i.e., it is an $S L(N, \mathbb{C})$-matrix if the gauge transformations take values in $S U(N)$. Gauge transformations act on $M$ via $M \rightarrow M^{g}=g M$, where $g$ is an element of the group $G$, say, e.g., $S U(N)$. Wave functions are gauge-invariant functionals of $H=M^{\dagger} M$, with the inner product given as

$$
\langle 1 \mid 2\rangle=\int d \mu(H) \exp \left[2 c_{A} S_{w z w}(H)\right] \Psi_{1}^{*} \Psi_{2}
$$

Here $S_{w z w}$ is the Wess-Zumino-Witten action (WZW), given by

$$
\begin{aligned}
S_{w z w}(H)= & \frac{1}{2 \pi} \int \operatorname{Tr}\left(\partial H \bar{\partial} H^{-1}\right) \\
& +\frac{i}{12 \pi} \int \epsilon^{\mu \nu \alpha} \operatorname{Tr}\left(H^{-1} \partial_{\mu} H H^{-1} \partial_{\nu} H H^{-1} \partial_{\alpha} H\right)
\end{aligned}
$$

In Eq. (2), $d \mu(H)$ is the Haar measure for $H$ which takes values in $S L(N, \mathbb{C}) / S U(N)$. Also $c_{A}$ denotes the value of the quadratic Casimir operator for the adjoint 
representation; it is equal to $N$ for $S U(N)$. The Hamiltonian and other observables can be expressed as functions of the current $J$ of the WZW action, namely,

$$
J=\frac{2}{e} \partial H H^{-1}
$$

(We have included a prefactor involving the coupling $e$; this is useful for later calculations.) The explicit formula worked out in Refs. [2-4] is given as $\mathcal{H}=\mathcal{H}_{0}+\mathcal{H}_{1}$, where

$$
\begin{aligned}
\mathcal{H}_{0}= & m \int_{z} J_{a}(\vec{z}) \frac{\delta}{\delta J_{a}(\vec{z})}+\frac{2}{\pi} \int_{z, w} \frac{1}{(z-w)^{2}} \frac{\delta}{\delta J_{a}(\vec{w})} \frac{\delta}{\delta J_{a}(\vec{z})} \\
& +\frac{1}{2} \int_{x}: \bar{\partial} J^{a}(x) \bar{\partial} J^{a}(x): \\
\mathcal{H}_{1}= & i e f_{a b c} \int_{z, w} \frac{J^{c}(\vec{w})}{\pi(z-w)} \frac{\delta}{\delta J_{a}(\vec{w})} \frac{\delta}{\delta J_{b}(\vec{z})}
\end{aligned}
$$

where $m=e^{2} c_{A} / 2 \pi$. Regularization issues have been discussed in some detail in the cited references.

The basic strategy we used was to solve the Schrödinger equation keeping all terms in $\mathcal{H}_{0}$ at the lowest order, treating $\mathcal{H}_{1}$ as a perturbation. In ordinary perturbation theory (carried out using our Hamiltonian formulation), one would expand $H=\exp \left(t_{a} \varphi^{a}\right)$ in powers of the Hermitian field $\varphi^{a}$; in addition, since $m=e^{2} c_{A} / 2 \pi$ we would also expand in powers of $m$. In our case, we keep the term involving $m$ even at the lowest order. So even if we expand $H$ in terms of $\varphi^{a}$, our expansion would correspond to a partially resummed version of what would be normal perturbation expansion. Formally, we keep $m$ and $e$ as independent parameters in keeping track of different orders in solving the Schrödinger equation, only setting $m=$ $e^{2} c_{A} / 2 \pi$ at the end. The lowest order computation of the wave function in this scheme was given in [3] and gave the string tension for a Wilson loop in the representation $R$ as $\sigma_{R}=e^{4} c_{A} c_{R} / 4 \pi, c_{R}$ being the quadratic Casimir value for the representation $R$. We have also considered corrections to this formula, taking the expansion to the next higher order (which still involves an infinity of correction terms) and found that the corrections were small, of the order of $-0.03 \%$ to $-2.8 \%$ [6]. The resulting values for the string tension agree well with the lattice estimates $[7,8]$.

Some of the other issues explored within this approach include string breaking effects [9], effective action and $\mathbb{Z}_{N}$ vortices [10], supersymmetric theories [11], and entanglement effects [12]. Glueball masses have been discussed in [13].

\section{THE CASIMIR ENERGY FOR PARALLEL WIRES}

There is an important feature which emerged from our analysis, which is very useful for the present purpose [14]. We can absorb the factor $e^{2 c_{A} S_{w z w}}$ in (2) into the definition of the wave function by writing $\Psi=e^{-c_{A} S_{w z w}} \Phi$. The inner product for the $\Phi$ 's will involve just the Haar measure without the $e^{2 c_{A} S_{w z w}}$ factor. However, the Hamiltonian acting on $\Phi$ will now be given by $\mathcal{H} \rightarrow e^{c_{A} S_{\text {wzw }}} \mathcal{H} e^{-c_{A} S_{\text {wzw }}}$. We can expand $H$ as $H=\exp \left(t_{a} \varphi^{a}\right) \approx 1+t_{a} \varphi^{a}+\cdots$, with the field $\varphi^{a}$ being hermitian. As mentioned earlier, this "small $\varphi$ " expansion is suitable for a (resummed) perturbation theory. The Hamiltonian is then

$$
\mathcal{H}=\frac{1}{2} \int\left[-\frac{\delta^{2}}{\delta \phi^{2}}+\phi\left(-\nabla^{2}+m^{2}\right) \phi+\cdots\right]
$$

where $\phi_{a}(\vec{k})=\sqrt{c_{A} k \bar{k} /(2 \pi m)} \varphi_{a}(\vec{k})$. This is clearly the Hamiltonian for a field of mass $m$ with the corresponding vacuum wave function

$$
\Phi_{0} \approx \exp \left[-\frac{1}{2} \int \phi^{a} \sqrt{m^{2}-\nabla^{2}} \phi^{a}\right]
$$

The Hamiltonian (6) corresponds to the action

$S=\int d^{3} x \frac{1}{2}\left[\dot{\phi}^{a} \dot{\phi}^{a}-\left(\nabla \phi^{a}\right)\left(\nabla \phi^{a}\right)-m^{2} \phi^{a} \phi^{a}\right]+\cdots$

These results show that the propagator for the gaugeinvariant component of the gluon field is the same as that of a massive scalar field with mass equal to $m=\left(e^{2} c_{A} / 2 \pi\right)$. Further, the parametrization (1) of the gauge potentials becomes, in the small $\varphi$-expansion

$$
\begin{aligned}
A_{i}^{a} & \approx \frac{1}{2}\left[-\partial_{i} \theta^{a}+\epsilon_{i j} \partial_{j} \varphi^{a}+\cdots\right], \\
M & =\exp \left(-\frac{i}{2} t_{a}\left(\theta^{a}+i \varphi^{a}\right)\right)
\end{aligned}
$$

In the case of a perfectly conducting plate, the boundary condition is that the tangential component of the electric field should be zero. In other words, we need

$$
\epsilon_{i j} n_{i} F_{0 j}^{a}=0
$$

where $n_{i}$ is the unit vector normal to the plate. This is also the condition used in [5]. In terms of the parametrization in (9), focusing just on the gauge-invariant part $\varphi^{a}$, this means that we need

$$
n_{i} \epsilon_{i j} \epsilon_{j k} \partial_{k} \dot{\varphi}^{a}=-n_{i} \partial_{i} \dot{\varphi}^{a}=0
$$

Since the time-derivative does not affect the spatial boundary conditions, this is equivalent to imposing Neumann boundary conditions on the scalar field $\varphi^{a}$ or, equivalently, on $\phi^{a}$. The end result is that, within this approximation of keeping $m$, but expanding the field $H$ to the lowest order in $\varphi^{a}$, the Casimir energy will be given by that of a massive 
scalar field with Neumann boundary conditions on the plates.

We now consider the standard arrangement of two parallel plates (or rather wires since we are in two spatial dimensions) which are of infinite extent in the $x_{2}$-direction and are normal to the $x_{1}$-direction. The wires are separated by a distance $R$. We take the range of $x_{2}$ to be $L$, with $L \rightarrow \infty$ eventually. The fields in the region between the wires have the mode expansion

$$
\phi^{a}=\int \frac{d k}{2 \pi} \sum_{n=0}^{\infty} C_{n, k}^{a} \sqrt{\frac{2}{R}} \cos \left(\frac{n \pi x_{1}}{R}\right) e^{i k x_{2}}
$$

This is consistent with the Neumann boundary conditions. We note that the Casimir energy of massive scalar fields for the parallel plate geometry with Dirichlet boundary conditions is known [15]. The result for Neumann conditions is essentially the same. Here we reproduce the result and express it in a form more suitable for comparison with lattice estimates. With the mode expansion (12), the action (8) becomes

$S=\int d t \frac{d k}{2 \pi} \sum_{n} \frac{1}{2}\left[\dot{C}_{n, k}^{a} \dot{C}_{n, k}^{a}-\Omega_{n, k}^{2} C_{n, k}^{a} C_{n, k}^{a}\right]+\cdots$

where $\Omega_{n, k}^{2}=k^{2}+(n \pi / R)^{2}+m^{2}$. The diagonalization of the Hamiltonian is trivial, yielding the unrenormalized zero-point energy

$$
\begin{aligned}
\mathcal{E} & =\frac{L}{2} \operatorname{dim} G \int \frac{d k}{2 \pi} \sum_{n} \sqrt{(n \pi / R)^{2}+k^{2}+m^{2}} \\
& =\frac{L}{2 \Gamma\left(-\frac{1}{2}\right)} \operatorname{dim} G \int \frac{d k}{2 \pi} \int_{0}^{\infty} \frac{d s}{s^{3 / 2}} e^{-s\left(k^{2}+m^{2}\right)} \sum_{n=0}^{\infty} e^{-s(n \pi / R)^{2}}
\end{aligned}
$$

Using the Poisson summation formula we get

$$
\begin{aligned}
\mathcal{E}= & \frac{L}{2 \Gamma\left(-\frac{1}{2}\right)} \operatorname{dim} G \int \frac{d k}{2 \pi} \int_{0}^{\infty} \frac{d s}{s^{3 / 2}} e^{-s\left(k^{2}+m^{2}\right)} \\
& \times \frac{1}{2}\left[1+\frac{R}{\sqrt{\pi s}}+2 \sum_{n=1}^{\infty} \frac{R}{\sqrt{\pi s}} e^{-n^{2} R^{2} / s}\right]
\end{aligned}
$$

The first two terms in this expression are divergent and they have to be subtracted. The first term is independent of the distance $R$ between the wires, corresponds to a self-energy contribution, and gets subtracted when we consider the energy shift $\mathcal{E}(R)-\mathcal{E}(R \rightarrow \infty)$, which is the relevant renormalized quantity of interest. The second term is proportional to the spatial volume $R L$ and is part of a uniform spatial density of vacuum energy which must also be subtracted out in the renormalized expression for the Casimir energy. The final renormalized expression is thus

$$
\begin{aligned}
\mathcal{E}= & \frac{L}{2 \Gamma\left(-\frac{1}{2}\right)} \operatorname{dim} G \int \frac{d k}{2 \pi} \int_{0}^{\infty} \frac{d s}{s^{3 / 2}} e^{-s\left(k^{2}+m^{2}\right)} \\
& \times \sum_{n=1}^{\infty} \frac{R}{\sqrt{\pi s}} e^{-n^{2} R^{2} / s}
\end{aligned}
$$

Doing the $k$-integration and using the variable transformation $s=(n R / m) e^{\theta}$, we find

$$
\begin{aligned}
\mathcal{E} & =-\frac{L R}{4} \operatorname{dim} G\left(\frac{m}{\pi R}\right)^{3 / 2} \sum_{n=1}^{\infty} \int \frac{d \theta}{n^{3 / 2}} \cosh (3 \theta / 2) e^{-2 n m R \cosh \theta} \\
& =-\frac{L R}{4} \operatorname{dim} G\left(\frac{m}{\pi R}\right)^{3 / 2} \sum_{n=1}^{\infty} \frac{K_{3 / 2}(2 n m R)}{n^{3 / 2}}
\end{aligned}
$$

Using the following expression for modified Bessel function $K_{3 / 2}$,

$$
K_{3 / 2}(z)=\sqrt{\frac{\pi}{2 z}} e^{-z}\left(1+\frac{1}{z}\right)
$$

we can rewrite the Casimir energy as

$\mathcal{E}=-\operatorname{dim} G \frac{L}{16 \pi R^{2}}\left[2 m R \operatorname{Li}_{2}\left(e^{-2 m R}\right)+\mathrm{Li}_{3}\left(e^{-2 m R}\right)\right]$

where $\operatorname{Li}_{s}(w)$ is the polylogarithm function

$$
\mathrm{Li}_{s}(w)=\sum_{1}^{\infty} \frac{w^{n}}{n^{s}}
$$

We may note that, in the $m \rightarrow 0$ limit, the expression (19) agrees with the well-known result for $(\operatorname{dim} G)$ massless scalars in $(2+1)$ dimensions,

$$
\mathcal{E}_{m=0}=-\operatorname{dim} G \frac{L \zeta(3)}{16 \pi R^{2}}
$$

There are other equivalent ways to arrive at result (19). Using

$$
\Omega_{n, k}=\left.\left(\frac{\partial^{2}}{\partial x_{0}^{2}}\right) \int \frac{d k_{0}}{\pi} \frac{e^{i k_{0} x_{0}}}{k_{0}^{2}+\Omega_{n, k}^{2}}\right|_{x_{0}=0}
$$

we can carry out the summation over $n$ (in (14)) to obtain

$$
\begin{aligned}
\mathcal{E} & =-L R^{2} \operatorname{dim} G \int \frac{d^{2} k}{(2 \pi)^{2}} \frac{k_{0}^{2}}{\omega} \frac{1}{e^{2 \omega}-1} \\
& =-\frac{L R}{4 \pi} \operatorname{dim} G \int_{0}^{\infty} d p \frac{p^{3}}{\sqrt{p^{2}+m^{2}}} \frac{1}{e^{2 R \sqrt{p^{2}+m^{2}}}-1}
\end{aligned}
$$

where $\omega^{2}=R^{2}\left(k_{0}^{2}+k^{2}+m^{2}\right)$ and in the second line we used polar coordinates in the $\left(k, k_{0}\right)$-plane and integrated 
over the angle and $p=\sqrt{k_{0}^{2}+k^{2}}$. A further substitution $p R=\sinh q$, and $z=\cosh q$, reduces this to

$$
\mathcal{E}=-\operatorname{dim} G \frac{L}{4 \pi R^{2}}(m R)^{3} \int_{1}^{\infty} d z \frac{\left(z^{2}-1\right)}{e^{2 m R z}-1} .
$$

Expansion of the integrand in powers of $e^{-2 m R z}$ gives the result (19) in terms of the polylogarithms.

It is useful to write the energy (19), for our case, in terms of the string tension corresponding to the fundamental representation. This has been calculated in [3]. Ignoring the small corrections discussed in [6], this is given by

$$
\sigma_{F}=e^{4} \frac{c_{A} c_{F}}{4 \pi} .
$$

We may thus write $m R=\sqrt{c_{A} / \pi c_{F}} x$, where $x=R \sqrt{\sigma_{F}}$. The Casimir energy is thus given by

$$
\begin{aligned}
\frac{\mathcal{E}}{L \sigma_{F}}= & -\frac{\operatorname{dim} G}{16 \pi}\left[\frac{2 \sqrt{c_{A} / \pi c_{F}}}{x} \operatorname{Li}_{2}\left(e^{-2 \sqrt{c_{A} / \pi c_{F}} x}\right)\right. \\
& \left.+\frac{1}{x^{2}} \operatorname{Li}_{3}\left(e^{-2 \sqrt{c_{A} / \pi c_{F}} x}\right)\right] .
\end{aligned}
$$

This is the main result of this paper. It holds for an arbitrary compact group; for the case of $S U(N)$, we have $c_{A}=N$, $c_{F}=\left(N^{2}-1\right) /(2 N)$. There will be corrections to this formula due to the fact that we have neglected interactions involving cubic and higher powers of $\varphi^{a}$ and due to the corrections to the string tension in the expression for $m$ in terms of $\sigma_{F}$. Nevertheless, the fact that string tension given in (25) to the lowest order in our expansion scheme is in good agreement with lattice calculations [7] suggests that the formula (26) can be a good estimate of the Casimir energy.

We have the Neumann boundary condition on the field $\phi^{a}$ for perfectly conducting wires, as mentioned before. But if we choose different boundary conditions, the result can be different. Formula (26) holds for the field obeying Neumann conditions at both wires or Dirichlet conditions at both wires. The Dirichlet condition is equivalent to the magnetic field $B$ (which is $-\nabla^{2} \varphi^{a}$ in our approximation) vanishing at the wire. If we consider the Neumann condition at one wire and the Dirichlet condition at the other, the modes involved are of the form $\sin \left(\left(n+\frac{1}{2}\right) \pi x_{1} / R\right)$. The Casimir energy is now given by

$\mathcal{E}_{D N}=\frac{L}{2} \operatorname{dim} G \int \frac{d k}{2 \pi} \sum_{n} \sqrt{\left(\left(n+\frac{1}{2}\right) \pi / R\right)^{2}+k^{2}+m^{2}}$.

The renormalized finite Casimir energy now works out to be

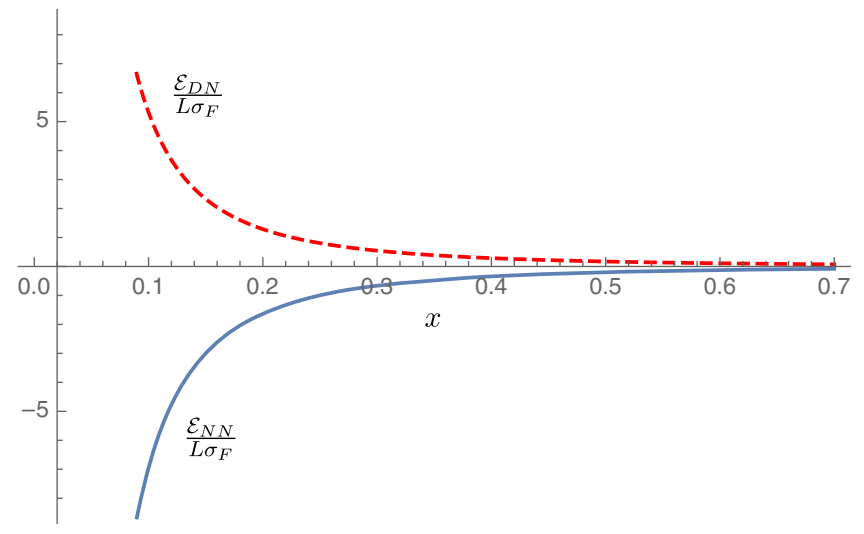

FIG. 1. Comparison of $\mathcal{E}_{N N}=\mathcal{E}_{D D}$ from (26) (solid line) and $\mathcal{E}_{D N}$ from (28) (dashed line).

$$
\begin{aligned}
\mathcal{E}_{D N}= & -\operatorname{dim} G \frac{L}{16 \pi R^{2}}\left[2 m R \operatorname{Li}_{2}\left(-e^{-2 m R}\right)+\operatorname{Li}_{3}\left(-e^{-2 m R}\right)\right] \\
\frac{\mathcal{E}_{D N}}{L \sigma_{F}}= & -\frac{\operatorname{dim} G}{16 \pi}\left[\frac{2 \sqrt{c_{A} / \pi c_{F}}}{x} \operatorname{Li}_{2}\left(-e^{-2 \sqrt{c_{A} / \pi c_{F}} x}\right)\right. \\
& \left.+\frac{1}{x^{2}} \operatorname{Li}_{3}\left(-e^{-2 \sqrt{c_{A} / \pi c_{F}} x}\right)\right] .
\end{aligned}
$$

Notice that, as expected, this corresponds to a repulsive force because the arguments of the polylogarithms have changed sign. The two energies $\mathcal{E}$ from (26) (same as $\mathcal{E}_{N N}=\mathcal{E}_{D D}$ ) and $\mathcal{E}_{D N}$ from (28) are shown in Fig. 1 for the case of $S U(2)$.

\section{LATTICE ESTIMATES AND THE MAGNETIC MASS}

The Casimir energy for the parallel wire geometry was recently evaluated for the $S U(2)$ gauge theory by lattice simulation in [5], with the boundary condition of the tangential component of the electric field vanishing at the wires. (This would be the Neumann-Neumann case for the field $\phi^{a}$ in our parametrization of $A_{i}^{a}$.) Essentially, the expectation value of the energy density was calculated, with a suitable renormalization. The result was fitted to the form

$$
\frac{\mathcal{E}}{L \sigma_{F}}=-\operatorname{dim} G \frac{\zeta(3)}{16 \pi} x^{-\nu} e^{-M_{\text {Cas }} x / \sqrt{\sigma_{F}}}
$$

The authors find that the best fit values of the parameters are $\nu=2.05$ and $M_{C a s}=1.38 \sqrt{\sigma_{F}}$. The authors also commented on the fact that $M_{C a s}$ is significantly smaller than the smallest value for glueball mass, which is approximately $4.7 \sqrt{\sigma_{F}}$. The smallness of the exponent is not a surprise from our point of view, since the coefficient of $x$ in the exponential in (26) is $2 \sqrt{c_{A} / \pi c_{F}}=2 \sqrt{8 / 3 \pi} \sim$ 1.84 for the case of $S U(2)$. This is also, as expected, significantly smaller than what is given by the glueball 
mass. While the numerical value differs from the value for $M_{\text {Cas }} / \sqrt{\sigma_{F}}$ in [5], it should be noted that the form of the function is different as well. The motivation to use (29) as a fitting function for the Casimir energy was that it reduced to the massless formula correctly, upon setting $M_{\text {Cas }}=0$ and $\nu=2$. So it may be viewed as a two-parameter extension of the formula for the massless case. Our formula (26) also correctly reduces to the massless limit, and so one may contemplate a modification of (26) with additional parameters to be used as a fitting function. One could consider, e.g., changing the prefactor in (26); there is a reasonable argument for this. Notice that the prefactor is a measure of the number of degrees of freedom, as evidenced by the $\operatorname{dim} G$ factor. Lattice simulations of QCD shows that the number of degrees of freedom do not quite reach a value corresponding to a gas of free gluons even at very high temperatures, where we expect a deconfined gluon plasma. (This has been known for a while; a recent review which gives updated results is [16]; in particular, see Fig. 4 of this reference.) In our calculation presumably such an effect can arise from higher-order terms in $\varphi^{a}$ which have been neglected.

Another observation is that our calculation based on the previous Hamiltonian analysis shows, as explained in more detail later, that there are strong theoretical reasons why the expression for the Casimir energy should involve powers of $e^{-2 m R}$ where $m$ is the mass as it appears in the propagator for the gauge-invariant part of the gauge potential. So here we will keep the value of $m$ as the one given by the Hamiltonian analysis, not treated as a parameter to be obtained from fitting. However, based on what was said earlier, we shall use a prefactor and try to fit the lattice calculation to the formula

$$
\frac{\mathcal{E}}{L \sigma_{F}}=-A \frac{\operatorname{dim} G}{16 \pi}\left[\frac{1.84}{x} \operatorname{Li}_{2}\left(e^{-1.84 x}\right)+\frac{1}{x^{2}} \operatorname{Li}_{3}\left(e^{-1.84 x}\right)\right]
$$

where $A$ is to be treated as fitting parameter and we have also put in the values of $c_{A}, c_{F}$ for $S U(2)$. A comparison of (29) and (30), with the best fit values $\nu=2.05, M_{C a s}=$ $1.38 \sqrt{\sigma_{F}}$ for (29) and $A=0.9715$ for (30), is shown in Fig. 2. The range of $x$ is taken to be 0.1 to 0.7 as done in [5]. The graph shows clearly that our formula does capture the lattice calculation of the Casimir energy with good quantitative accuracy.

It is worth emphasizing the significance of the gaugeinvariant Hamiltonian analysis we have used here. A priori, it is not clear that the Casimir effect for the non-Abelian theory can be reduced to that of a massive scalar field. Our approach shows that this can indeed be done. Second, we get a specific value for the propagator mass $m$, namely, $e^{2} c_{A} /(2 \pi)$, as well as its relation to the string tension, since we also have an independent prediction for $\sigma_{F}$. Taking this value, without determining it via fitting to lattice data, we get good agreement. We have used an overall coefficient $A$

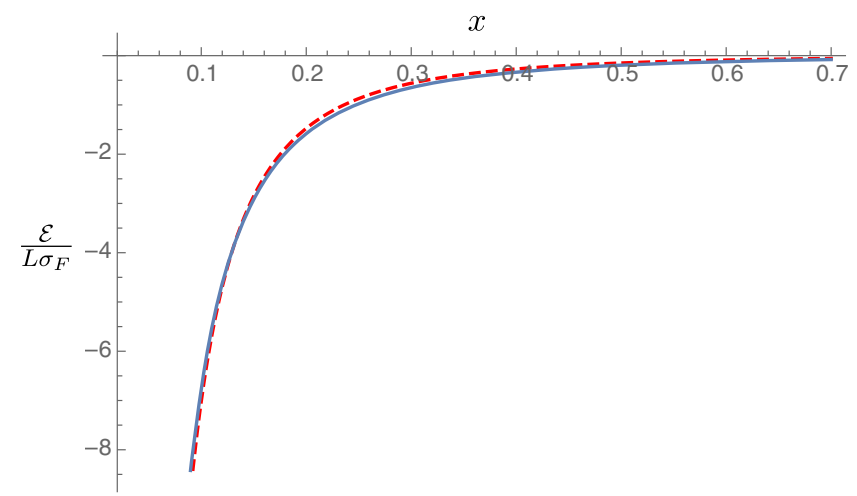

FIG. 2. Comparison of (29) (dashed line) and (30) (solid line).

as a parameter determined by fitting. But the best fit value is 0.9715 , so that in retrospect, we see that even if we took $A$ to be 1 , as it is in our lowest order calculation, the agreement is still within a few percent.

The good agreement between the lattice results in [5] and our analytical expression (26) for the Casimir energy provides yet another strong indication (in addition to the string tension agreement $[7,8]$ ) that our Hamiltonian analysis, in particular the quadratic approximation, provides a good effective description for $(2+1)$-dimensional Yang-Mills theory.

The mapping of the Casimir energy to that of a massive scalar field has been discussed in [17] $]^{1}$ for compact Abelian electrodynamics in three dimensions, where the monopoles are responsible for the mass generation. Our approach justifies a mapping to the massive scalar for the nonAbelian Yang-Mills theory, and also yields predictions for $m$ and $\sigma_{F}$.

A few more comments on the formula for the Casimir energy are in order at this point. First of all, there is an intuitive reasoning for the exponential dependence on $x$ which is as follows. The expectation value of the energy involves the propagator since

$$
\left.\langle\mathcal{E}\rangle \sim \int \frac{\partial}{\partial x_{0}} \frac{\partial}{\partial x_{0}^{\prime}}\left\langle A_{i}^{a}\left(\vec{x}, x_{0}\right) A_{i}^{a}\left(\vec{x}, x_{0}^{\prime}\right)\right\rangle\right|_{x_{0}=x_{0}^{\prime}}+\cdots
$$

The propagator $\left\langle A_{i}^{a}\left(\vec{x}, x_{0}\right) A_{i}^{a}\left(\vec{x}, x_{0}^{\prime}\right)\right\rangle$ may be viewed in terms of paths from $\vec{x}$ to one of the wires, from there to the other wire, and then back to $\vec{x}$. This involves a distance of $2 R$, and with a propagator mass of $m$, we expect a factor $e^{-2 m R}$. This should hold for all boundary conditions for large $R$. Multiple transits can lead to the formula with the summation as in the polylogarithm. This argument, as well as our explicit calculation, makes it clear that the mass in the propagator is what controls the exponential factor. Of course the precise functional dependence of the Casimir energy on $e^{-2 m R}$ depends on the boundary conditions, as displayed e.g., in Eqs. (26) and (28).

\footnotetext{
${ }^{1}$ We thank M. Chernodub for bringing these papers to our attention.
} 
Second, we note that the propagator mass is also related to the magnetic screening mass in one higher dimension. If we consider the $(3+1)$-dimensional Yang-Mills theory at very high temperatures, in the imaginary time formalism, all modes except for the lowest Matsubara frequency decouple and the theory is expected to reduce to a threedimensional one with $e^{2}=g^{2} T$, where $g$ is the 4-d coupling and $T$ is the temperature. The mass which appears in the propagator of the Euclidean 3-dimensional theory then serves as the magnetic screening mass of the high temperature $(3+1)$-dimensional theory. For this reason, we often refer to the propagator mass in our calculation [which is $m$ in the quadratic approximation, as mentioned after Eq. (8)] as the magnetic mass. We are only considering the pure Yang-Mills case here. In a theory like QCD, we have fermions as well; since they do not have a mode of zero Matsubara frequency (due to the antiperiodicity condition) they do not survive the dimensional reduction at high temperatures. Thus the propagator mass of the Euclidean 3-dimensional theory will serve as the magnetic mass in the approximation of neglecting corrections due to fermions.

As mentioned earlier, the functional dependence of the Casimir energy on $m$ will depend on the choice of boundary conditions and, more generally, on the geometry of the configuration used. Thus fitting the energy to a single exponential for different setups can yield different values for what might be considered a screening mass or the $M_{\text {Cas }}$ as in [5]. Any attempt to make a direct identification of this with the magnetic mass can be ambiguous. The point is that the use of the Hamiltonian (5) or (6) will involve only the single parameter $m$, with different formulas for the different configurations. If the formula appropriate to the setup is used, all measurements should lead to a consistent value for $m$; it is this value which can be identified with the magnetic mass (modulo the fermionic contribution mentioned earlier).
As for the values of the magnetic mass, our Hamiltonian calculation gives $m=e^{2} c_{A} / 2 \pi=e^{2} / \pi \approx 0.32 e^{2}$, for $S U(2)$. There have been many other ways of estimating the magnetic mass. These include various resummation and gap equation approaches [18-21], lattice analyses in different gauges [22] and a method of identifying the magnetic mass as a common divisor for glueball masses [23]. The values obtained are close to what we find, generally in the range 0.28 to 0.38 for $m / e^{2}$, with the lattice values being somewhat higher, close to 0.5 . There is general consistency among the values, none of them is close to the glueball masses. (All numerical values with a short discussion are given in [4].) Since there is some variation, one could also envisage the mass as a fitting parameter, although our experience with the string tension suggests that the Hamiltonian approach should be closest to lattice simulations.

Finally, the main point which emerges from this discussion is that the Casimir effect in the $(2+1)$-dimensional theory is a good probe of the magnetic mass for the $(3+1)$ dimensional theory, in the limit of neglecting corrections due to fermions, keeping in mind that the formula derived from (6) for the chosen geometry should be used to estimate the mass parameter. Therefore, lattice simulations for groups other than $S U(2)$ and comparison with our formula (26), [or (28) for mixed boundary conditions] with perhaps a prefactor $A$ to be determined, will be worthwhile, in terms of providing greater insights into this issue.

\section{ACKNOWLEDGMENTS}

We thank Hans Hansson for bringing the paper [5] to our attention. We also thank Maxim Chernodub for discussions. This research was supported in part by the U.S. National Science Foundation Grant No. PHY-1519449 and by PSC-CUNY awards.
[1] See, e.g., D. Gross, R. Pisarski, and L. Yaffe, Rev. Mod. Phys. 53, 43 (1981) and references therein.

[2] D. Karabali and V. P. Nair, Nucl. Phys. B464, 135 (1996); Phys. Lett. B 379, 141 (1996).

[3] D. Karabali, C. Kim, and V. P. Nair, Nucl. Phys. B524, 661 (1998); Phys. Lett. B 434, 103 (1998).

[4] For a short review, see V. P. Nair, in Workshop on QCD Green's Functions, Confinement and Phenomenology, QCD-TNT, Trento, Italy (2009); Proc. Sci., POS (QCDTNT09) (2009) 030 [arXiv:0910.3252].

[5] M. N. Chernodub, V. A. Goy, A. V. Molochkov, and H. H. Nguyen, arXiv:1805.11887 [Phys. Rev. Lett. (to be published)].

[6] D. Karabali, V. P. Nair, and A. Yelnikov, Nucl. Phys. B824, 387 (2010).
[7] M. Teper, Phys. Rev. D 59, 014512 (1998) and references therein.

[8] B. Lucini and M. Teper, Phys. Rev. D 66, 097502 (2002); B. Bringoltz and M. Teper, Phys. Lett. B 645, 383 (2007).

[9] A. Agarwal, D. Karabali, and V. P. Nair, Nucl. Phys. B790, 216 (2008).

[10] V. P. Nair, Phys. Rev. D 85, 105019 (2012).

[11] A. Agarwal and V. P. Nair, Phys. Rev. D 85, 085011 (2012); J. Phys. A 48, 465401 (2015).

[12] A. Agarwal, D. Karabali, and V. P. Nair, Phys. Rev. D 96, 125008 (2017).

[13] R. G. Leigh, D. Minic, and A. Yelnikov, Phys. Rev. Lett. 96, 222001 (2006); Phys. Rev. D 76, 065018 (2007).

[14] D. Karabali and V. P. Nair, Phys. Rev. D 77, 025014 (2008). 
[15] P. Hays, Ann. Phys. (N.Y.) 121, 32 (1979); J. Ambjorn and S. Wolfram, Ann. Phys. (N.Y.) 147, 1 (1983); See also M. Bordag, U. Mohideen, and V. M. Mostepanenko, Phys. Rep. 353, 1 (2001); K. A. Milton, The Casimir Effect: Physical Manifestations of Zero Point Energy, Lectures at 17th Symposium on Theoretical Physics, Seoul National University, Korea (1998); M. V. Cougo-Pinto, C. Farina, and A. J. Segui-Santonja, Lett. Math. Phys. 31, 309 (1994); S. Mobassem, Mod. Phys. Lett. A 29, 1450160 (2014).

[16] R. Pasechnik and M. Sumbera, Universe 3, 7 (2017).

[17] M. N. Chernodub, V. A. Goy, and A. V. Molochkov, Phys. Rev. D 95, 074511 (2017); 96, 094507 (2017).

[18] J. M. Cornwall, Phys. Rev. D 10, 500 (1974); 26, 1453 (1982); 57, 3694 (1998); J. M. Cornwall, W. S. Hou, and J. E. King, Phys. Lett. 153B, 173 (1985); J. M. Cornwall and
B. Yan, Phys. Rev. D 53, 4638 (1996); J. M. Cornwall, Phys. Rev. D 57, 3694 (1998); 76, 025012 (2007).

[19] V. P. Nair, Phys. Lett. B 352, 117 (1995); G. Alexanian and V. P. Nair, Phys. Lett. B 352, 435 (1995).

[20] W. Buchmuller and O. Philipsen, Nucl. Phys. B443, 47 (1995); O. Philipsen, TFT-98: Thermal Field Theories and their Applications, edited by U. Heinz, arXiv:hep-ph/ 9811469; R. Jackiw and S.-Y. Pi, Phys. Lett. B 442, 291 (1998).

[21] R. Jackiw and S. Y. Pi, Phys. Lett. B 368, 131 (1996).

[22] F. Karsch, T. Neuhaus, A. Patkós, and J. Rank, Nucl. Phys. B474, 217 (1996); F. Karsch, M Oevers, and P Petreczky, Phys. Lett. B 442, 291 (1998).

[23] O. Philipsen, Phys. Lett. B 521, 273 (2001); arXiv:hep-lat/ 0110114. 